\title{
Costing clinical biochemistry services as part of an operational management budgeting system
}

\author{
IF TARBIT \\ From the Department of Clinical Biochemistry, Freeman Hospital, Newcastle upon Tyne
}

SUMMARY The process of costing clinical biochemistry tests as a component of the commissioning of a unit management budgeting system based on an International Computers Limited (ICL) minicomputer system was examined. Methods of apportioning consumable and labour costs under direct and indirect cost headings and as test and request charges were investigated, and in this currently operational system it was found that $38 \%$ of consumable costs and $57 \%$ of labour costs were not a direct component of the routine analysis function.

Means of assigning test costs to a given request source and the incorporation of such charges into clinical budget statements were looked at. A reduction in laboratory workload did not produce a comparable reduction in laboratory costs. For a theoretical reduction in workload of $20 \%$ only a $3.8 \%$ laboratory saving in recoverable costs could be expected.

After the publication of the Griffiths Report management budgeting systems were started in several centres. With the object of monitoring and controlling use of resources, procedures are continually being devised to cost the use of services by the clinician end user and provide him or her with regular budget information.

After the initial pilot studies of management budgeting in $1983^{1}$ Newcastle Health Authority asked Arthur Young, McClelland, Moors and Co (AYMM) to set up a management budgeting system at Freeman Hospital, Newcastle upon Tyne, using ICL International Computers Limited DRS (Distributed Resource System) computer hardware.

The clinical biochemistry department at Freeman Hospital ( 720 beds, including a 180 bed regional cardiothoracic unit) is staffed by a consultant chemical pathologist, senior registrar, top grade biochemist, three other biochemists, and 21 medical laboratory scientific officers. Workload in 1985 was 171259 requests, which generated 775559 tests.

The AYMM team arrived on site in August 1984. Their brief was to create suitable costing procedures and management budgeting mechanisms and achieve an operational package by April 11985 . To show the system's ability to promote cost effective patient care a small group of eight clinicians participated during the pilot stages as end users. After the system became operational the number of clinicians receiving budget statements and given guidance in their interpretation gradually increased, and continues to do so.

Accepted for publication 10 March 1986

\section{Costing procedures}

AYMM consultants contacted the pathology departments in September 1984 and requested the provision of a full range of analysis costs by the end of October 1984. For the clinical biochemistry department, this entailed the production of over 170 individual costings within about eight weeks. For certain groups of analysis probably performed on more than one instrument, as with the serum electrolyte profile processed on SMA 6/60 or Beckman Astra analysers, each instrument profile had to be costed separately. As well as composite costings for electrolyte, bone and liver, and cardiac profiles, costings had to be assessed for each analysis within the profile that might be requested individually. Although there are perhaps 12-14 analyses that are regularly requested "on-call" and are accepted as part of the clinical biochemistry emergency service, there are a further group of analyses that could, in certain circumstances, be clinically justifiable as an emergency request-for example, serum iron or plasma digoxin. Any analysis of this nature also required costing on an emergency basis.

To minimise the extra work burden placed on any one person each section head (senior medical laboratory scientific officer or senior biochemist) was given the task of assessing consumable and reagent usage rates and labour time entailed in each analytical procedure within his or her section. Helped by the senior chief medical laboratory scientific officer, each section head then assigned consumable and reagent costs per 
test based on known costs from purchasing records. The senior chief medical laboratory scientific officer and top grade biochemist then checked each completed costing.

\section{DIRECT CONSUMABLE COSTS}

For automated equipment we saw the simple equation of theoretical tests per hour with number of samples processed per hour as overly simplistic for several reasons:

1 The speed of analysis of any automated instrument in tests per hour does not equate with the true sample throughput rate; calibration standards and quality control sera occupy a major proportion of "sample" positions in any analysis run.

2 Repeat analysis may be required if a particular batch of tests are "out of control", or instrument or power failure occurs.

3 The consumption of reagents can be severely affected by unproductive instrument start up, shut down, and stand by periods.

We assessed the true consumable costs over four months for each analysis or group of analyses by considering the actual number of sample analyses completed for any given volume of reagent. For each reagent and consumable the total cost to the department over the given period was divided by the number of samples analysed and reported in that period - to arrive at a cost per sample for the analysis procedure. Built in to these reagents costs per sample, therefore, are the cost of calibration, the cost of running quality control material, repeat analyses, and purging of instrument lines.

For small batches of manual analysis costs were assigned on the basis of the size of the average batch. Thus if the average batch of serum "rhubarb" analysis contains 12 samples, four calibrants, and three quality control samples then the total consumables costs for running these 19 tests is assigned to the 12 samples to determine the consumable cost per sample. All consumable costs must be incorporated into each costing, including, for example, pump tubing, dialysis membranes, and printer paper on automated procedures and pipette tips, plastic disposables, and filter paper for manual assays.

\section{DIRECT LABOUR COSTS}

Given the time scale of the exercise, we were not able to carry out the more detailed assessments of analytical time performed in previous costing studies. ${ }^{23}$ As factors such as laboratory housekeeping, staff training, machine maintenance, coffee breaks, answering telephone enquiries all cut into the theoretical time available for sample analysis, it was decided that six hours per working day per member of $\underline{\vec{\sigma}}$ staff was a realistic time available for analysis. Labour time for analysis procedures could be assessed most easily with automated analysis, particularly where one or more members of staff were fully committed to a particular analysis group throughout the working day. Thus for the bone and liver profile 35000 test profiles were performed in 1984 over 260 working days, giving an average sample throughput of 140 profiles per working day. This analysis profile was performed by one basic grade medical laboratory scientific officer for whom the available analysis time per day was $6 \times 60$ minutes (360 minutes). Thus for one bone and liver profile labour time was $360 / 140$ minutes-that is, 2.6 minutes at basic grade medical laboratory scientific officer labour rates.

For smaller batches of analyses the section head $\vec{v}$ was asked to assess a realistic time for analysis of the 음 average size batch, including reagent, calibrant, and quality control preparation, drawing up of work- $\rightleftarrows$ sheets and result reporting. This time per average batch was divided by the number of samples (not $\stackrel{5}{9}$ tests) in the average batch to determine the average $\vec{A}$ time per sample.

We were concerned that supervision of routine analysis should be seen as a direct labour charge on tests. It was agreed with AYMM consultants that a certain percentage of senior staff time should be allocated to direct costs and spread equally across each test under senior staff control. Similarly, vetting and validating reported results was regarded as a direct charge on tests, and an appropriate cost element for participating senior staff labour was assigned.

Table 1 shows the labour rates per minute of working time for each grade of medical laboratory scientific officer. These were calculated by the treasurer's department in the manner outlined. Changes in labour costs resulting from national pay awards and revised working conditions will, of course, necessitate adjustment on a regular basis.

To apply the then current on call rate of $£ 8.33$ to each emergency request received would be grossly $\frac{D}{0}$ excessive when more than one emergency request is received on the same emergency call out. We there- $N$ fore decided to calculate an average labour cost per $N$ request as the most reasonable approach to charging $\mathcal{N}$ for on call services, by dividing the total cost of $\omega$ emergency clinical biochemistry services for 1984 ?

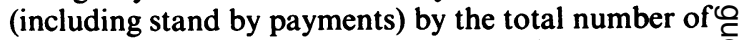
emergency requests for 1984, giving a labour cost per $\Phi$ emergency request of $£ 4.06$. Obviously, this charge + will vary according to the average number of requests per call (currently $2 \cdot 28$ ).

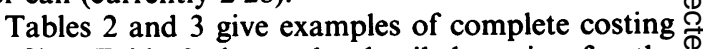
profiles. Table 2 shows the detailed costing for the $\varrho$ electrolyte profile on the SMA 6/60, which, with its 
Table 1 Calculation of labour rate per unit time

\begin{tabular}{|c|c|c|c|}
\hline Time per year available & $\begin{array}{l}\text { Basic grade medical laboratory } \\
\text { scientific officer }\end{array}$ & Time (days) & \\
\hline Days $=260$ & & $\begin{array}{l}\text { Annual holidays } \\
\text { Public holidays }\end{array}$ & $\begin{array}{l}=20 \\
=10 \\
\frac{30}{30}\end{array}$ \\
\hline $\begin{array}{l}\text { Working days }=260-30 \\
\text { Working minutes } \\
\quad(7 \cdot 4 \text { hour working day) }=230 \times 7.4 \times 60=102120 \\
\text { Mid point salary scale for grade } \\
\text { (including employer's contributions) }\end{array}$ & $=£ 7597$ & $=230$ & \\
\hline Labour rate per minute $=\frac{7597}{102120}$ & $=£ 0.074$ & & \\
\hline \multirow[t]{2}{*}{$\begin{array}{l}\text { Further modifying factors: Allowance for illness } \\
\text { Average day release for grade }\end{array}$} & & $\begin{array}{l}5 \\
7 \cdot 5\end{array}$ & \\
\hline & & $\overline{12 \cdot 5}$ & \\
\hline Adjustable available working time $=230-12 \cdot 5$ & & $\begin{array}{l}=217.5 \text { days } \\
=96750 \text { minutes }\end{array}$ & \\
\hline Adjusted labour rate per minute $=\frac{7597}{96750}$ & $=£ 0.079$ & & \\
\hline
\end{tabular}

Labour rates per unit time

\begin{tabular}{|c|c|c|c|}
\hline Grade & Rate/minute (£) & Grade & Rate/minute (£) \\
\hline \multirow{3}{*}{$\begin{array}{l}\text { Junior medical laboratory scientific officer } \\
\text { Junior/basic medical laboratory scientific } \\
\text { officer } \\
\text { Basic medical laboratory scientific officer } \\
\text { Basic/senior medical laboratory scientific } \\
\text { officer }\end{array}$} & 0.063 & \multirow{3}{*}{$\begin{array}{l}\text { Senior medical laboratory scientific officer } \\
\text { Senior or chief medical laboratory } \\
\text { scientific officer } \\
\text { Chief medical laboratory scientific officer }\end{array}$} & 0.097 \\
\hline & $\begin{array}{l}0.071 \\
0.079\end{array}$ & & $\begin{array}{l}0.106 \\
0.114\end{array}$ \\
\hline & & & \\
\hline
\end{tabular}

Based on 1985 salary figures before pay settlement.

Table 2 Standard cost calculation-electrolyte profile (SMA)

\begin{tabular}{ll}
\hline Consumables & \\
\hline Item & Cost per sample (f) \\
\hline Lithium sulphate & 0.0093 \\
Propane gas & 0.0013 \\
Chloride diluent & 0.0047 \\
Chloride colour reagent & 0.0067 \\
Carbon dioxide diluent & 0.0030 \\
Carbon dioxide colour reagent & 0.0112 \\
Urea acid reagent & 0.0026 \\
Urea colour reagent & 0.0042 \\
Creatinine sodium hydroxide reagent & 0.0017 \\
Creatinine sodium chloride reagent & 0.0016 \\
Creatinine colour reagent & 0.0024 \\
Calibration serum & 0.0496 \\
Quality control serum & 0.0295 \\
Phasing serum & 0.0032 \\
Pump tubing & 0.0149 \\
Dialysis membranes & 0.0034 \\
Computer paper & 0.0005 \\
Teletype labels & 0.0205 \\
Total consumables & 0.1703 \\
\hline
\end{tabular}

\begin{tabular}{llll}
\hline \multicolumn{3}{c}{ Labour } \\
\hline Grade & $\begin{array}{l}\text { Rate/ } \\
\text { minute }\end{array}$ & Minutes & Cost (£) \\
\hline $\begin{array}{l}\text { Senior medical laboratory } \\
\text { scientific officer }\end{array}$ & 0.097 & 2.0 & 0.194 \\
$\begin{array}{l}\text { Basic medical laboratory } \\
\text { scientific officer }\end{array}$ & 0.079 & 2.0 & 0.158 \\
$\begin{array}{c}\text { Consultant/registrar/ } \\
\text { scientist* }\end{array}$ & & 0.050 \\
$\begin{array}{l}\text { Total labour } \\
\text { Total unit rate }=\mathbf{£ 0 . 5 7}\end{array}$ & & 0.40 \\
\hline
\end{tabular}

*Element of cost incorporated for vetting and validation of reports.

high throughput and low reagent costs, is relatively cheap compared with the cost of paraprotein investigation by immunoelectrophoresis (Table 3), a complex manual investigation using costly reagents and therefore considerably more expensive. 
Table 3 Standard cost calculation-immunotyping by immunoelectrophoresis

\begin{tabular}{ll}
\hline Consumables & \\
\hline Item & Cost per sample $(\mathfrak{})$ \\
\hline Electrophoresis buffer & 0.043 \\
Sample tips & 0.050 \\
Protein stain & 0.010 \\
Stain remover & 0.016 \\
Agarose electrophoresis plate & 1.350 \\
STA-moist paper & 0.213 \\
Antisera & 1.060 \\
Total consumables & $2 \cdot 740$ \\
\hline
\end{tabular}

\begin{tabular}{llll}
\hline Labour & & & \\
\hline Grade & $\begin{array}{l}\text { Rate/ } \\
\text { minute }\end{array}$ & Minutes & Cost $(£)$ \\
\hline $\begin{array}{l}\text { Basic/senior medical } \\
\text { laboratory scientific } \\
\text { officer }\end{array}$ & 0.088 & 60.0 & 5.28 \\
$\begin{array}{l}\text { Consultant/registrar/ } \\
\text { scientist }\end{array}$ & & 0.05 \\
$\begin{array}{l}\text { Total labour } \\
\text { Total unit rate }=£ 8.07\end{array}$ & & 5.33 \\
\hline
\end{tabular}

Direct costs relating to requests rather than tests also had to be incorporated into the system. Essentially, this concerned the costs of receiving and separating samples before analysis. Consumables used in the sample reception area and their costs were determined over four months, and the consumable cost per sample received was determined by dividing the total cost of each consumable over the four months by the number of samples processed in that period. Similarly, the total labour time over that period was divided by the number of samples processed to determine labour time (and hence costs) per sample. This gave a total request cost for sample handling of $£ 0 \cdot 22$, which was incorporated into a blanket overhead cost per request.

\section{INDIRECT COSTS}

It was recognised that the income generated from the total direct cost of tests would not entirely recoup the departmental budget. There are two possible approaches to full recovery:

1 A uniform overhead charge per request that incorporates the indirect costs of running the department.

A proportion of each senior member of staff's salary was seen as an indirect cost and incorporated into this general request cost and reflected the costs incurred in departmental management, provision of advice and consultation services to clinicians, research and method development functions, and education and training functions. In addition, the sample handling charge of $£ 0.22$ per request had been assigned to recovery under this heading. Table 4 details the cost elements incorporated in this overhead charge. It is the intention that medical staff salaries and hotel and general service charges will soon be charged to departmental budgets and will eventually appear under this overhead charge per request.

2 Once a full year's costing data are available, the extent to which the total consumables budget for the department is recovered as direct costs of tests can be assessed.

Any shortfall from total recovery can be corrected by applying a percentage uplift factor based on this shortfall to each test's total consumables costs, so that for the following year the total consumables budget will be recovered in full as direct costs of tests. Similarly, once the first year's costs have been

Table 4 Derivation of request costs and charges

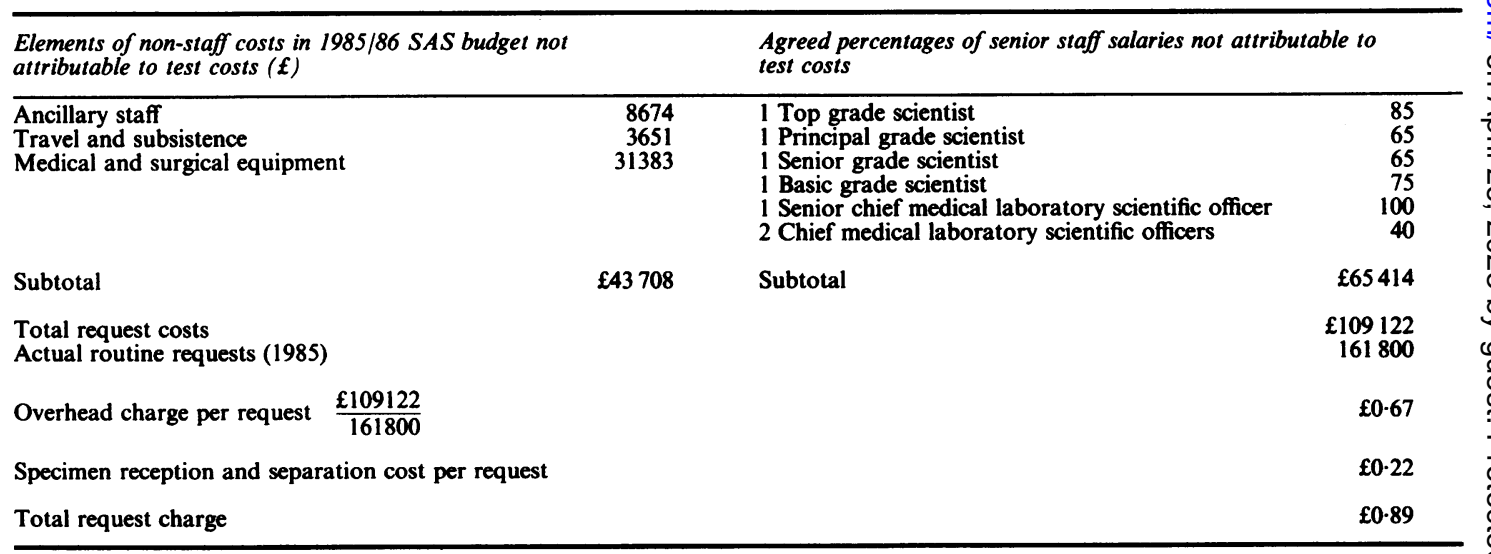

SAS = NHS standard accounting system 
Test Rate List

Department: Freeman Biochemistry

$\begin{array}{lr}\text { Test } & \text { Test } \\ \text { Code } & \text { Rate } \\ 011 & 0.5\end{array}$

0110.5700

$012 \quad 1.0400$

$013 \quad 0.6931$

$014 \quad 1.7200$

$015 \quad 0.9500$

$016 \quad 0.2800$

$017 \quad 1.4600$

$018 \quad 2.0700$

$019 \quad 2.2600$

$020 \quad 0.2900$

$2 \cdot 5600$

$1 \cdot 2700$

1.5800

1.8800

0.2500

0.3000

$4 \cdot 2300$

3.0200

0.2700

0.6600

3.5200

0.2900

0.4600

0.4300

2.4400

0.2600

0.4400

$4 \cdot 8400$

3.3200

1.5200

1.6400

0.4100

0.2700

0.5100

0.8600

0.8600

5.4400

0.4300

0.8400

10.5500

0.4500

0.3000

3.9500

1.3500

0.8000

0.6600

$2 \cdot 4500$

1.5000

0.4500

0.2700

0.2700

0.5100

1.3700

3.4000

7.6500

4.5300

$10 \cdot 1100$

0.2700

0.5100

2.4500

2.8800

0.2600

0.4700

4.0000

Urea
Description

Urea and electrolytes profile (SMA)

Urea and electrolytes profile (ASTRA)

Bone and liver profile and AST

Cardiac enzyme profile, CK + CK - MB

Acid phosphatase

Albumin (SMA)

Alcohol

Albumin manual

Alpha fetoprotein

Alkaline phosphatase

Alkaline phosphatase isoenzymes

Amylase

Acid glycoproteins

Antitrypsin

Aspecitate aminotransferase, (AST)

Bilirubin

Bilirubin (direct)

Caeruloplasmin

Calcium (SMA)

Calcium (atomic absorption)

lonised calcium

$\mathrm{CO}_{2}$ content (SMA)

$\mathrm{CO}_{2}$ content (ASTRA)

Carboxyhaemoglobin

Carotenoids

Chloride (SMA)

Chloride (ASTRA)

Cholesterol, triglyceride

Cholinesterase

Copper

Cortisol

Creatine kinase, (CK)

Creatinine (SMA)

Creatinine (ASTRA)

Cryoglobulins

Complement C3, C4

Differential protein clearance

Glucose (AAII)

Glucose (ASTRA)

Glucose 6PDH

Gamma glutamyl transferase (GT)

HBDH

High density lipoprotein cholesterol

Iron, Iron binding capacity

Lipoprotein electrophoresis

Magnesium

Methaemoglobin

Methaemalbumin

Osmolality

Phosphate inorganic

Potassium (SMA)

Potassium (ASTRA)

Protein total manual

Protein electrophoresis

Protein immunoelectrophoresis

Protein IgG, IgM, IgA

Protein immunofixation

Sodium (SMA)

Sodium (ASTRA)

Sulphaemoglobin

Transferrin

Urea (ASTRA)

Uric acid

Xylose
Test Rate List

Department: Freeman Biochemistry

$\begin{array}{lr}\text { Test } & \text { Test } \\ \text { Code } & \text { Rate } \\ 087 & 1.5\end{array}$

$087 \quad 1.5200 \quad$ Zin

$089 \quad 0.2700 \quad$ Protein total SMA

$090 \quad 2.6400 \quad$ RIA-Digoxin

RIA-T3 uptake

$092 \quad 0.9900 \quad$ RIA-T4

$093 \quad 5.3200 \quad$ RIA-Ferritin

$094 \quad 1.9600 \quad$ RIA-TSH

$2.9800 \quad$ RIA $-F S H$, LH

2.9500 RIA-TSH, T4

3.5000 RIA-T3

4.4900 RIA-Microglobulin

0.3100 Urine-Albumin

$2.0400 \quad$ Urine-Amylase (diastase)

1.5000

0.8100

$2 \cdot 0400$

$2 \cdot 6000$

0.6800

$1 \cdot 1000$

$2 \cdot 0100$

0.3900

0.3800

0.7500

0.8100

$3 \cdot 8300$

0.4100

0.4100

8.9500

0.4500

0.2900

0.3500

0.6500

0.5900

$1 \cdot 7400$

0.9300

0.3300

0.5900

5.0100

0.6100

1.3500

$2 \cdot 3800$

$3 \cdot 3500$

2.0400

0.3100

$5 \cdot 3800$

7.0800

0.9000

0.4300

0.2700

0.4300

1.6900

3.7500

$7 \cdot 5000$

0.4200

0.3700

$4 \cdot 7700$

$2 \cdot 4500$

$4 \cdot 4000$

4.4900

0.6200

$2 \cdot 9500$

1. 6300

Urine-Bilirubin

Urine-Calcium

Urine-Copper

Urine-Cortisol

Urine-Creatinine

Urine-5HIAA

Urine-HMMA

Urine-Homogentisic acid

Urine-Hypobromite

Urine-Indican

Urine-Magnesium

Urine-Metadrenaline

Urine-Melanin

Urine-Myoglobin

Urine-Oxalate

Urine-Osmolality

Urine-pH

Urine-Phosphate

Urine-Protein quantitative

Urine-Potassium

Urine-Porphyrin

Urine-Reducing substances

Urine-Specific gravity

Urine-Sodium

Urine-Transferrin

Urine-Urea

Urine-Uric acid

Urine-Urobilinogin

Urine-Xylose

Urine-Zinc

Urine-Protein

Urine-Protein electrophoresis

Urine-PABA Excretion

Urine-Electrolytes

Miscellaneous fluid-Glucose

Miscellaneous fluid-Protein

CSF-Glucose

CSF-Protein

CSF-Globulin

Faeces_Fat balance

Faeces - Occult blood

Faeces-Trypsin

Renal calculi

Drugs_Lithium

Drugs-Methotrexate

Drugs-Paracetamol

Drugs - Rifampicin

Drugs - Quinidine

Drugs-Salicylate

Drugs - Theophylline

Fig. 1 Full test rate listing showing numerical test code and unit rates (f).SMA = Technicon SMA 6/60-Vickers SP120 multichannel analyser; $A S T R A=B e c k m a n$ ASTRA multichannel analyser; $A A I I=$ Technicon AAII analyser. 
(b)

Pathology tests: CLIN BIOCHEMISTRY

Printed: 05/10/85

Pathology tests: CLIN BIOCHEMISTRY Report month 09

Centre code and description
Test code and description
326 Ward 26-Cardiothoracic Surgery
011 Urea and electrolytes (prof) (SMA)
012 Urea and electrolytes (prof) (ASTRA)
014 Cardiac profile, CK + MB
062 Magnesium
138 Urine-electrolytes
328 Ward 27-Cardiothoracic Surgery
011 Urea and electrolytes (SMA)
012 Urea and electrolytes (ASTRA)
013 Bone and liver profile \& AST
044 CK
052 Glucose (AAlI)
141 Misc fluid-protein
212 Urea and electrolytes (prof) (ASTRA)
(on call emergency request)
231 Calcium atomic absorption
(on call emergency request)
253 Glucose Astra
(on call emergency request)
262 Magnesium
(on call emergency request)
270 Protein total
(on call emergency request)

\begin{tabular}{lll}
$\begin{array}{l}\text { No of } \\
\text { tests }\end{array}$ & $\begin{array}{l}\text { Unit } \\
\text { rate }\end{array}$ & Value \\
& & \\
1 & 0.5700 & 1 \\
3 & 1.0400 & 3 \\
4 & 1.7200 & 7 \\
3 & 0.6600 & 2 \\
3 & 0.9000 & 3 \\
\hline 14 & & $\frac{16}{14}$
\end{tabular}

$0.5700 \quad 1$

1.04002

$0.6931 \quad 1$

0.4100

$0.4300 \quad 1$

0.2700

$4 \cdot 8100 \quad 10$

$4 \cdot 2400 \quad 4$

$4.6100 \quad 5$

$4 \cdot 2400 \quad 4$

$\frac{1}{16} \quad 4 \cdot 4100 \quad \frac{4}{32}$

Pathology tests: CLIN BIOCHEMISTRY

Report month 09

Printed: 05/10/85 Centre code and description
Test code and description

512 A N Surgeon Cardio-surgery In patient 011 Urea and electrolytes (SMA) 012 Urea and electrolytes (ASTRA) 013 Bone and liver profile \& AST 014 Cardiac enzyme profile, CK + CK - MB 12 016 Albumin (SMA) 018 Albumin manual 032 lonised calcium 039 Cholesterol, triglyceride 044 Creatine kinase 052 Glucose (AAII)

058 Iron, iron binding capacity

066 Osmolality

070 Protein total manua

120 Urine-Osmolality

138 Urine-Electrolytes

212 Urea and electrolytes (ASTRA) (on call emergency request)

253 Glucose Astro (on call emergency request) 266 Osmolality (plasma) (on call emergency request) 308 Urine-Creatinine (on call emergency request) 324 Urine-Potassium (on call emergency request) 328 Urine-Sodium (on call emergency request) 330 Urine-Urea (on call emergency request)

Unit Value

$\begin{array}{lll}142 & 0.5700 & 81\end{array}$

$1.0400 \quad 16$

0.693160

$1.7200 \quad 21$

$0.2800 \quad 1$

2.07002

$3.5200 \quad 4$

$4.8400 \quad 10$

$0.4100 \quad 4$

$0.4300 \quad 3$

$1.3500 \quad 1$

0.4500

$1.3700 \quad 1$

$0.4500 \quad 1$

0.900021

$4.8100 \quad 63$

$4.6100 \quad 5$

$\begin{array}{lll}1 & 4.2500 \quad 4\end{array}$

$1 \quad 4 \cdot 2800 \quad 4$

$1 \quad 4.2800 \quad 4$

$\frac{1}{326} \quad 4 \cdot 2100 \frac{4}{314}$

513 A N Surgeon-Cardio-surgery Out patient 011 Urea and electrolytes (prof) (SMA) 013 Bone and liver profile \& AST 044 CK
$4 \cdot 2000 \quad 4$
Centre code and description

Test code and description

109 Nursing services

120 Biochemistry

126 Occupational therapy

300 Ward 1-urology surgery

301 Ward 2-urology surgery

302 Ward 3-urology surgery

303 Ward 4-nephrology

304 Ward 5-general/dental surgery

305 Ward 6-dermatology

306 Ward 7

307 Ward 8-general/dental surgery

308 Ward 9-cardiology

309 Ward 9a-cardiololgy

310 Ward 10-general medical and geriatrics

342 General ITU

343 Emergency Admission Suite

346 Out patients-main department

522 Con A-cardiology Out patient

524 Con B-cardiology In patient

525 Con B - cardiology Out patient

527 Con C-cardiology In patient

548 Con D-chest medicine-In patient 549 Con D-chest medicine-Out patient

575 Con E-Gen med \& geriatrics - In patient

576 Con E-Gen med \& geriatrics-Out patient

578 Con F-Gen med \& geriatrics - In patient

579 Con F-Gen med \& geriatrics-Out patient

602 Con G-nephrology-In patient

603 Con G-nephrology-Out patient

(c)

Pathology tests: CLIN BIOCHEMISTRY

Report month 09

\section{Centre code and description}

Test code and description

011 Urea and electrolytes (SMA)

012 Urea and electrolytes (ASTRA)

013 Bone and liver profile \& AST

014 Cardiac enzyme profile. CK + CK - MB $363 \quad 1.7200 \quad 627$

015 Acid phosphatase

016 Albumin (SMA)

017 Alcohol

018 Albumin manual

019 Alpha feto protein

021 Alkaline phosphate isoenzymes

023 Amylase

024 Acid glycoproteins

025 Antitrypsin

029 Caeruloplasmin

031 Calcium (atomic absorption)

032 lonised calcium

036 Carotenoids

039 Cholesterol, triglyceride
Printed: 05/10/85

Total Total

11

303

5
18

27

12

68

20

1
10

118

103

191

78

54

439

43

288

41

693

172

801

013

1218

Printed: 05/10/85

Unit Value

$0.5700 \quad 2967$

$.0400 \quad 474$

$0.9500 \quad 69$

$0.2800 \quad 4$

$1.4600 \quad 43$

$2.0700 \quad 4$

$2.2600 \quad 29$

$2.5600 \quad 153$

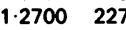

$1.5800 \quad 39$

$1.8800 \quad 10$

3.02003

$\begin{array}{lll}0.6600 & 17\end{array}$

$3.5200 \quad 244$

2.44002

$4.8400 \quad 1120$

\begin{tabular}{ccc}
3 & 0.5700 & 2 \\
4 & 0.6931 & 3 \\
1 & 0.4100 & \\
\hline 8 & & 5
\end{tabular}

Fig. 2 Specimen section of monthly printout of workload related to request source showing: (a) breakdown of test workload and charges (f) related to request cost centre (wards and clinicians); (b) summary of total analysis requests and charges (f) to each cost centre; (c) summary of total numbers of each test. SMA = Technicon SMA 6/60 - Vickers SP120 multichannel analyser; ASTRA = Beckman ASTRA multichanne analyser; $A A I I=$ Technicon $A A I I$ analyser $; C O N=$ Consultant . 


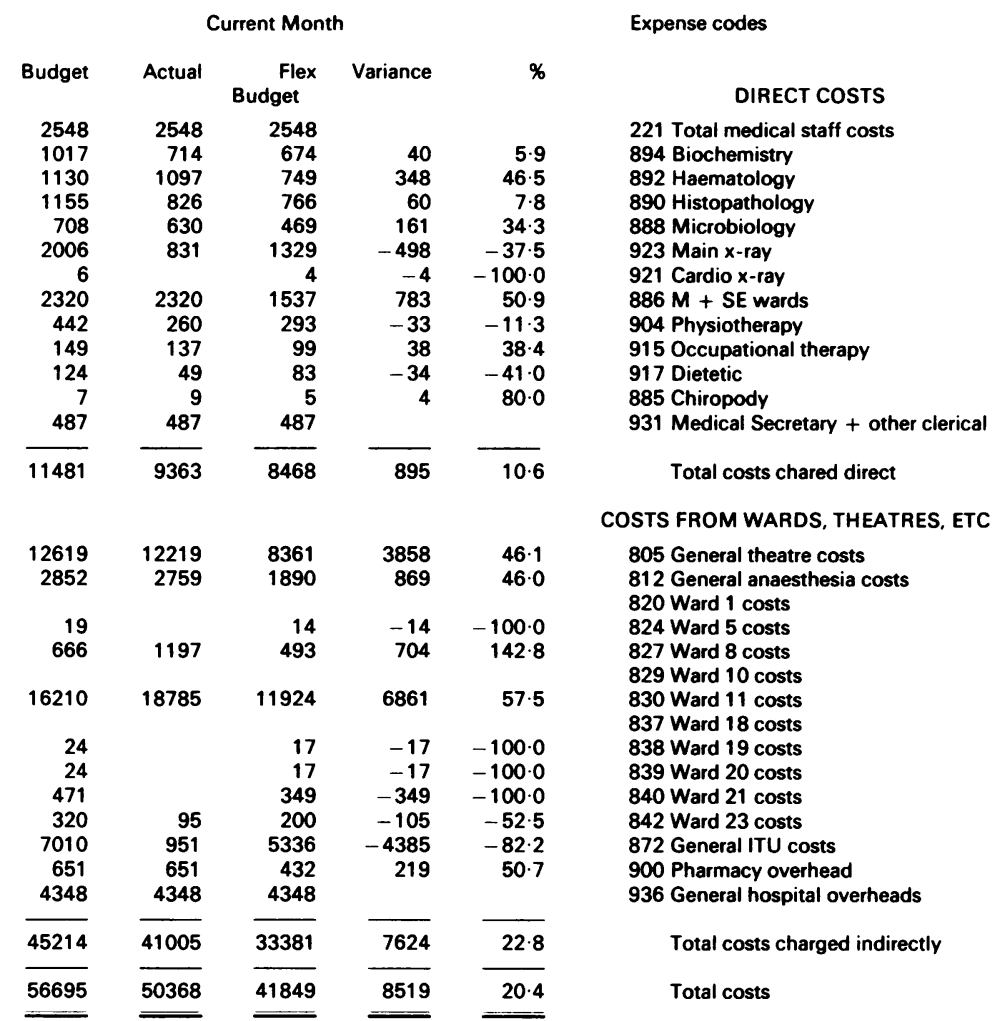

\begin{tabular}{|c|c|c|c|c|}
\hline \multicolumn{5}{|c|}{ Year to Date } \\
\hline Budget & Actual & $\begin{array}{l}\text { Flex } \\
\text { Budget }\end{array}$ & Variance & $\%$ \\
\hline $\begin{array}{r}7644 \\
3051 \\
3390 \\
3465 \\
2124 \\
6018 \\
18 \\
6960 \\
1326 \\
447 \\
372 \\
21 \\
1461\end{array}$ & $\begin{array}{r}7644 \\
2732 \\
3256 \\
2586 \\
1963 \\
4525 \\
\\
6961 \\
817 \\
338 \\
98 \\
12 \\
1461\end{array}$ & $\begin{array}{r}7644 \\
2335 \\
2593 \\
2652 \\
1625 \\
4603 \\
14 \\
5324 \\
1015 \\
343 \\
287 \\
17 \\
1461\end{array}$ & $\begin{array}{r}397 \\
663 \\
-66 \\
338 \\
-78 \\
-14 \\
1637 \\
-198 \\
-5 \\
-189 \\
-5\end{array}$ & $\begin{array}{r}17.0 \\
25.6 \\
-2.5 \\
20.8 \\
-1.7 \\
-100.0 \\
30.7 \\
-18.5 \\
-1.5 \\
-65.9 \\
-29.4\end{array}$ \\
\hline 34443 & 30822 & 28148 & 2674 & $9 \cdot 5$ \\
\hline $\begin{array}{r}37857 \\
8556\end{array}$ & $\begin{array}{r}42731 \\
9657 \\
25\end{array}$ & $\begin{array}{r}28953 \\
6545\end{array}$ & $\begin{array}{r}13778 \\
3112 \\
25\end{array}$ & $\begin{array}{l}47.6 \\
47.5\end{array}$ \\
\hline $\begin{array}{r}57 \\
1998\end{array}$ & $\begin{array}{r}2893 \\
287\end{array}$ & $\begin{array}{r}47 \\
1645\end{array}$ & $\begin{array}{r}-47 \\
1248 \\
297\end{array}$ & $\begin{array}{r}100.0 \\
75.9\end{array}$ \\
\hline 48630 & $\begin{array}{r}54517 \\
27\end{array}$ & 40788 & $\begin{array}{r}13729 \\
27\end{array}$ & $33 \cdot 7$ \\
\hline $\begin{array}{r}72 \\
72 \\
1413 \\
860 \\
21030 \\
1953 \\
13044\end{array}$ & $\begin{array}{r}602 \\
570 \\
2139 \\
1953 \\
13044\end{array}$ & $\begin{array}{r}57 \\
57 \\
1165 \\
683 \\
17646 \\
1496 \\
13044\end{array}$ & $\begin{array}{r}-57 \\
-57 \\
-563 \\
-113 \\
-15507 \\
457\end{array}$ & $\begin{array}{r}-100.0 \\
-100.0 \\
-48.3 \\
-16.5 \\
-87.9 \\
30.5\end{array}$ \\
\hline 135642 & 128455 & 112126 & 16329 & $14 \cdot 6$ \\
\hline 170085 & 159277 & 140274 & 19003 & 13.5 \\
\hline
\end{tabular}

Fig. 3 Specimen monthly budget statement for consultant surgeon.

assessed, that proportion of labour costs recovered as part of direct costs of tests can be ascertained. This figure can be compared with the proportion of staff costs, which, it was agreed, are recoverable in the form of charges on direct costs. This includes all medical laboratory scientific officer salaries below the level of chief medical laboratory scientific officer plus a proportion of each senior member of staff's salary, which, it was agreed, would be a direct charge on tests for supervisory roles of routine analysis functions. This total labour charge to be assigned to test costs will exceed the labour costs actually recovered from test costs because of two factors: no cost element has as yet been allowed for the supervisory role of senior staff; of the 7.4 hour working day, six hours had been estimated as the time available for actual analysis, and 1.4 hours of work time per day therefore remain unaccounted for.

Again, any shortfall in recovery of labour costs seen as a charge on test costs will be overcome by applying a percentage uplift factor to adjust the labour charge of each test, so that full recovery is achieved.

\section{DATA INPUT}

Each costed test or test profile was identified by a three figure numerical code. Fig. 1 shows a specimen section of the test rate listing. A similar series of three figure numerical codes was used to identify each cost centre location. Thus each consultant had two codes, one for inpatients and one for outpatients. Each ward and outpatient department also had its own specific code, which permits analyses to be charged against the patient's ward if the consultant is not known. Similarly, other departments and hospitals within the district or region that use Freeman Hospital biochemical services were also provided with a code. For each request for biochemistry analysis received by the department the appropriate cost centre code was written on to the analysis worksheet with the patient details. After analysis the test code and its associated cost centre code-that is, source of request-were input via the VDU's to the computer data base from the worksheets, with the laboratory generated patient identification number. At the end of each month a summary of analyses, requests, and request sources were printed out on the matrix printer situated in the 
clinical biochemistry department. The printout follows the format of:

1 Tests requested by ward and consultant and costs incurred.

2 Summary of total number of tests and requests and costs for each cost centre.

3 Summary of total numbers of each test or test profile.

Fig. 2 gives relevant examples.

The ICL DRS computer network system installed to run the management budgeting system uses a "stand alone" pathology network based on a DRS model 50 minicomputer system with 180 kilobyte random access memory and dual 27 megabyte sectioned hard disc files, which means that each department within pathology can access and sort its own data independently. The monthly summary of cost data is downloaded on to 8 inch floppy discs, which are passed to administration to be reloaded on to their Model 50 minicomputer linked to a 27 megabyte disc. The DRS model 50 in pathology is linked to two DRS model 10 "intelligent" VDU's and a 120 character per second matrix printer in the clinical biochemistry department. Radiology also has its own stand alone minicomputer from which information is transferred to the administration system via floppy discs. Costing data from other service departments, such as occupational therapy, dietetics, catering etc are loaded into the administration minicomputer through the associated terminal once a week from daily worksheets.

The unit accountant responsible to the unit general manager will collate this information and produce the monthly budget spread sheets for clinicians and service departments. These budget statements detail costs of services actually used against the allocated budgetary figure for that service, based on expected patient treatment rates. Where the actual patient treatment rate differs from the expected treatment rate, the budget figure is "flexed" to provide a new target figure. The difference or variance in actual expenditure from the flexed budget figure represents an efficiency indicator. A negative variance reflects efficiency gains in terms of provisions of a range of services for less expenditure. Fig. 3 shows a specimen budget statement. Where planned savings accrue through positive management action a percentage return for reuse will be negotiable with the unit general manager, as will virement between budget headings by a cost centre head.

\section{Discussion}

The initial data collection system had several problems: the software was unable to merge patient files with the same laboratory identification number; disc storage was limited (16 megabytes for the whole hospital system), necessitating repeated culling of the data base to avoid disc storage saturation, resulting in data sort and print programmes becoming inoperative; data sort and print processes could only be initiated for the pathology department as a whole and not for individual departments, so that recovery of information was excessively laborious.

The revised version of the system allows each department in pathology to access and sort its own files independently and allows the user to record test data, update requests as more test information becomes available, and printout request reports. It should be noted that this is not a laboratory data management system, nor does it claim to be. Manual input of test data is the only option, and no interfacing to automated instruments is envisaged. This factor militates against the use of the data base for recording test results in departments with large workloads.

Within the time scale of the exercise we were unable to consider the detailed timings of analysis suggested by Broughton and Hogan ${ }^{2}$ and Stilwell. ${ }^{3}$ Despite the less sophisticated approach to assessment of labour time used in this study a comparison of direct labour times arrived at in the present exercise with those of Broughton and Hogan shows broad comparability (Table 5). Appreciable differences sometimes did occur, and these could have been due to improvements in methodology and instrumentation over the past four years.

Stilwell's methodology incorporates both direct and indirect costs in a total cost per test, in contrast to that of Broughton and Hogan where indirect costs are assigned uniformly to each request as a "handling charge". As Broughton and Hogan state their approach facilitates comparison of direct costs of different analytical methods or laboratories without distorting relativities or masking the direct cost components.

The AYMM system developed at the Freeman Hospital incorporates elements of both systems. A major proportion of overhead costs are applied as a uniform charge per request, thus avoiding undue distortion of relativities in test costs. A certain proportion of indirect labour costs, however, are assigned as a charge on tests.

Using the annual workload per test in 1985 , total direct cost of each test in that year was determined using the unit cost rates per test currently in operation (Table 6). Forty three per cent of consumable costs and $67 \%$ of labour costs were seen as indirect when considering the routine analysis function alone. Including consumable and labour cost of sample reception and separation, the corresponding figures were $38 \%$ and $57 \%$, respectively. Broughton and 
Table 5 Direct labour time per test

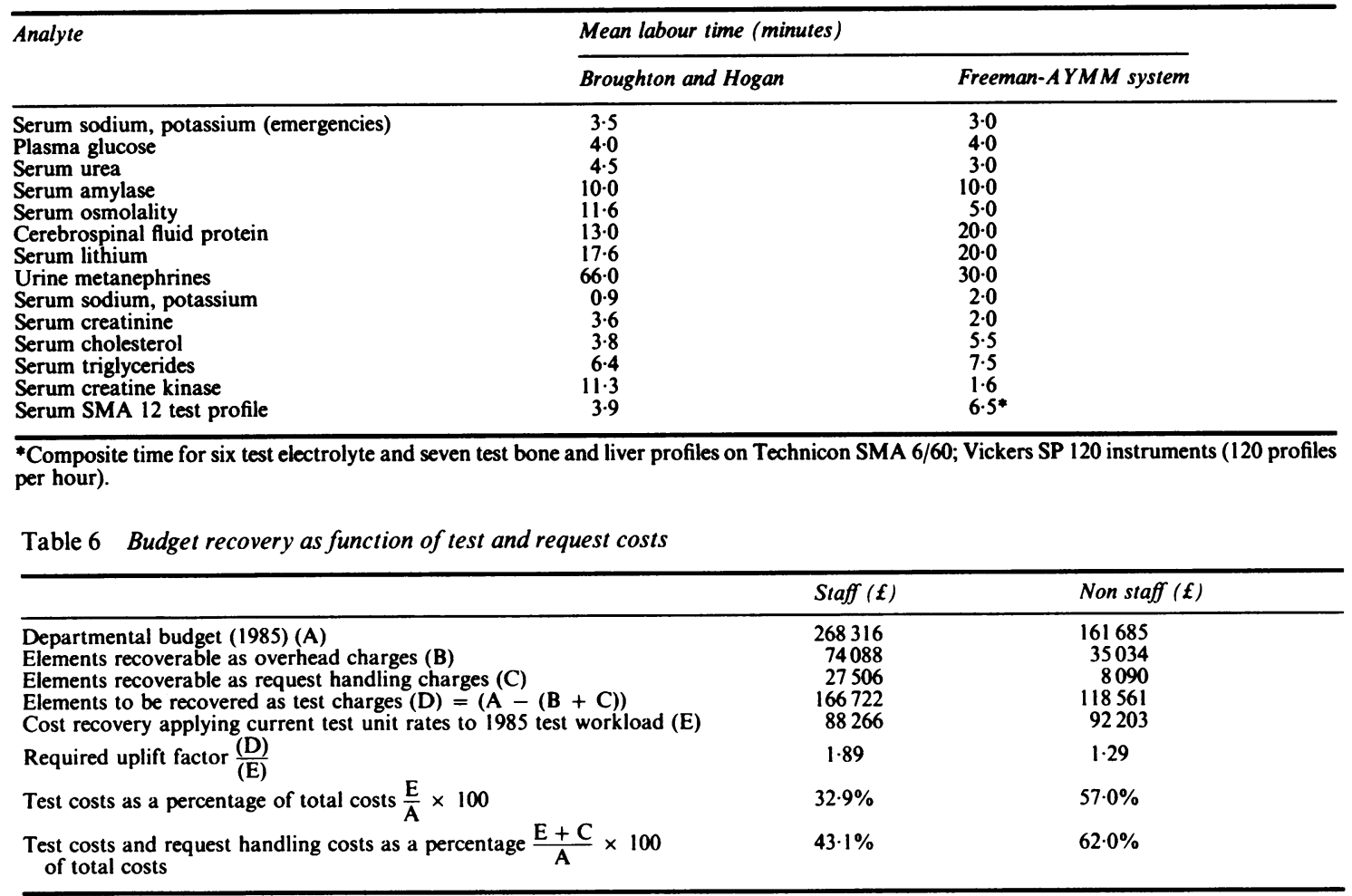

Table 7 Consumables as a percentage of total cost

\begin{tabular}{|c|c|c|c|c|c|}
\hline Test & $\begin{array}{l}\text { Total cost (£) } \\
\text { including request } \\
\text { charge }(£ 0.89)\end{array}$ & $\begin{array}{l}\text { Test consumables } \\
\text { (actual) as a percentage } \\
\text { of current total cost }\end{array}$ & $\begin{array}{l}\text { Total cost }(£) \text { with } \\
\text { uplifted consumables } \\
(29 \%) \text { uplifted labour } \\
(89 \%) \text { and request } \\
\text { charge }(\mathrm{f} 0.89)\end{array}$ & $\begin{array}{l}\text { Test consumables } \\
\text { (actual) as a } \\
\text { percentage of } \\
\text { revised total } \\
\text { cost }\end{array}$ & $\begin{array}{l}\text { Test and test } \\
\text { profile workload } \\
\text { per annum (1985) }\end{array}$ \\
\hline \multirow{2}{*}{$\begin{array}{l}\text { Electrolyte profile (SMA) } \\
\text { Electrolyte profile (ASTRA) } \\
\text { Bone and liver profile (SMA) } \\
\text { Cardiac enzyme profile } \\
\text { (creatine kinase } \\
\text { (CK) + CK-MB isoenzyme) }\end{array}$} & $\begin{array}{l}1.46 \\
1.93 \\
1.58\end{array}$ & $\begin{array}{l}11 \cdot 6 \\
38.9 \\
19 \cdot 2\end{array}$ & $\begin{array}{l}1.87 \\
2.41 \\
2.02\end{array}$ & $\begin{array}{r}9 \cdot 1 \\
31 \cdot 2 \\
15 \cdot 0\end{array}$ & $\begin{array}{r}55719 \\
8329 \\
34374\end{array}$ \\
\hline & $2 \cdot 61$ & $37 \cdot 3$ & 3.56 & 27.4 & 3719 \\
\hline $\begin{array}{l}\text { Creatine kinase } \\
\text { Glucose (AAII) } \\
\text { Amylase } \\
\text { Iron and iron binding capacity } \\
\text { Digoxin } \\
\text { Thyroxine } \\
\text { Thyroid stimulating hormone } \\
\text { Urine electrolytes (SMA) }\end{array}$ & $\begin{array}{l}1 \cdot 30 \\
1 \cdot 32 \\
2 \cdot 16 \\
2 \cdot 24 \\
3 \cdot 53 \\
1 \cdot 88 \\
2 \cdot 85 \\
1 \cdot 79\end{array}$ & $\begin{array}{r}17 \cdot 5 \\
4 \cdot 6 \\
26 \cdot 3 \\
13 \cdot 7 \\
46 \cdot 8 \\
27 \cdot 8 \\
42 \cdot 9 \\
12 \cdot 5\end{array}$ & $\begin{array}{l}1.52 \\
1.67 \\
2.95 \\
3.27 \\
4 \cdot 89 \\
2.45 \\
3.87 \\
2.48\end{array}$ & $\begin{array}{r}15.0 \\
3.6 \\
19.3 \\
9.4 \\
33.8 \\
21.3 \\
31.6 \\
9.0\end{array}$ & $\begin{array}{r}5753 \\
10417 \\
2427 \\
2187 \\
2681 \\
5942 \\
5986 \\
2167\end{array}$ \\
\hline Mean & 2.05 & 24.9 & 2.75 & $18 \cdot 8$ & $\begin{array}{l}93 \cdot 1 \% \text { of total test } \\
\text { workload }\end{array}$ \\
\hline
\end{tabular}

Hogan found $57 \%$ of consumable costs and $74 \%$ of labour costs to be indirect. Stilwell's study suggested that $52 \%$ of costs could be seen as indirect.

By calculating the shortfall between direct costs calculated from annual workload for 1985 and the proportion of annual budget that can be assigned under test costs, percentage uplift factors can be cal- culated to uniformly adjust consumables and labour portions of each test cost for full recovery of budget. Table 7 shows that uplift factors of 1.89 for labour and 1.29 for consumables applied to test costs would achieve full budget recovery.

Stilwell's study (1981) shows an average total cost per test of $£ 1.91$ for a range of 60 analytes, with $75 \%$ 
of these costs being less than or equal to $£ 2 \cdot 00$. At the Freeman Hospital in 1985 the mean cost total was $£ 3.19$, with $76 \%$ being less than or equal to $£ 4.00$ for a range of 66 regularly requested tests. For that group of 12 tests or test profiles, which comprise $93 \%$ of the laboratory workload, the average total cost per test or test profile is $£ 2.05$ at the time of writing. Applying uplift factors of 1.89 and 1.29 for labour and consumables, respectively, would increase the average total cost from $£ 2.05$ to $£ 2.75$ (Table 7).

Stilwell, Broughton and Hogan, and others ${ }^{4-6}$ incorporate capital cost elements into their costing systems, and a DHSS working party on costing activity in pathology departments in 1983 recommended that capital costs should be included in the aggregation of test costs. ${ }^{7}$ The AYMM system developed at Freeman Hospital, however, is designed to recover each service department's annual budget as income charged to users of services. Annual provision for capital replacement is not incorporated into NHS departmental budgets at present and is therefore not incorporated into the Freeman-AYMM system. The current value of capital equipment whose replacement value equals or exceeds $£ 1000$ in the clinical biochemistry department at Freeman Hospital amounts to $£ 440000$. Based on amortisation over ten years this would add $£ 44000$ to the current annual budget of $£ 430000$ - that is, an enhancement of $10 \%$ to be passed on as test costs to the user. On a cost per test basis the lack of incorporation of capital depreciation does not therefore unduly distort test costings in favour of automated tests as opposed to manual tests.

Heads of departments need to be aware of the possibility of simplistic deductions, which might be made once laboratory costing data are freely available. If budgetary constraints on clinicians lead them to radically reassess their need for clinical biochemistry services, resulting in, to take an extreme case, a $20 \%$ reduction in laboratory workload, every effort must be made to impress on unit general managers and unit accountants that this does not mean a $20 \%$ cut in costs incurred by the laboratory.

For those tests or test profiles that generate $93 \%$ of the clinical biochemistry workload at Freeman Hospital, the mean percentage of total costs per test that can be designated as consumable costs and therefore, to some extent, recoverable with reduction in workload is $24.9 \%$ (Table 7 ). Thus for a $20 \%$ reduction in income consequent on a $20 \%$ reduction in workload, only $24.9 \%$ of this - that is, $5 \%$-is a budgetary saving. Even this saving may be diminished by loss of discounts on bulk reagent purchases in certain cases. Adjusting current test costs by uplift factors to maximise recovery of laboratory budget reduces direct consumables contribution to an average of $18.8 \%$ of total cost per test, which would produce only a $3.8 \%$ budgetary saving with a $20 \%$ reduction in workload. Lundberg and Westlake ${ }^{8}$ observed this lack of direct response of laboratory operating costs to fluctuating workload to be one of the major elements which might lead, paradoxically, to increased charges per test if laboratory workload fell dramatically. In a detailed financial examination of an American laboratory Winkelman ${ }^{9}$ showed that a $10 \%$ reduction in workload would lead to a mere $1.8 \%$ reduction in direct costs for high volume automated tests and only a $4.9 \%$ reduction in direct test costs overall.

In a subsequent paper Winkelman and $\mathrm{Hill}^{10}$ outlined various strategies for coping with reduced funding consequent on declining workload. Of the strategies suggested, optimisation of the cost effectiveness of internal laboratory procedures without degradation of provision of services seems to be the most appropriate option for United Kingdom laboratories. It should be noted that the DHSS circular HN (85) 3 (1) (DHSS health notice (85), January 1985) on management budgeting recognises that reduction of the use of diagnostic tests will not lead to savings in manpower and overhead elements of test costs. Nevertheless, the onus is on laboratory staff to emphasise this vital point.

The AYMM system provides a valuable cost analysis tool that permits the identification of fluctuations in the variety and the number of requests per clinical team, changes in the overall volume of work per test, and the effects of these variables on costs incurred by the laboratory. The generation of an awareness of the relative cost of test procedures in the light of prevailing budgetary restrictions encourages rigorous laboratory assessment of the cost effectiveness of any new or modified analytical procedure in relation to its clinical utility.

In the post Griffiths era management budgeting systems are inevitably becoming widespread. The importance of the AYMM system lies in its current application as a working system rapidly being adopted by many hospitals in the United Kingdom. It is therefore in the interests of every head of a pathology department to understand the financial workings of his or her own department and to be aware of the elements that should be incorporated into a laboratory costing system. The cost of implementing management budgeting systems for a new site is estimated at $£ 90000-£ 100000$, with annual running costs totalling $£ 25000$ per unit (DHSS health notice (85), January 1985). It would therefore be naive not to assume that revenue savings will be expected to recover this expenditure.

At present, the management budgeting process is directed at the clinician end user, but eventually, close scrutiny must be given to laboratory budgets, particu- 
larly as performance indicators are refined and applied more extensively. Each laboratory head of department must therefore be prepared (with detailed costing information) to correct any misinformed ideas and convince unit and district general managers, when necessary, of the need to maintain and improve laboratory budgets. Detailed and thorough cost analysis of a laboratory's functions can only strengthen the case.

I am indebted to Mr C Patterson and Mr D Percival of Arthur Young, McClelland, Moors and Co, for many useful and interesting discussions over the past year, and to Mrs Sylvia Watson and Miss Monica Evans for valuable secretarial help.

\section{References}

${ }^{1}$ Pratt RA. A microcomputer based system for clinical budgeting. British Journal of Healthcare Computing 1984;1:23-8.

${ }^{2}$ Broughton PMG, Hogan TC. A new approach to the costing of clinical laboratory tests. Ann Clin Biochem 1981;18:330-42.
${ }^{3}$ Stilwell JA. Costs of a clinical chemistry laboratory. J Clin Pathol 1981:34:589-94.

${ }^{4}$ Krieg AF, Israel M, Fink R, Shearer LK. An approach to cost analysis of clinical laboratory services. Am J Clin Pathol 1978;69:525-36.

${ }^{5}$ Barnard DJ, Bingle JP, Garratt CJ. Cost of carrying out clinical diagnostic tests. $\mathrm{Br}$ Med J 1978;i:1463-6.

${ }^{6}$ Chapman C, Hayter C. Cost of carrying out clinical diagnostic tests. Br Med J 1978;ii:830.

' Fabray CE. Methods of measuring work and costing activity in pathology departments. NHS Financial Information Project Working Paper No 83/01. London: HMSO, 1983.

${ }^{8}$ Lundberg GD, Westlake GE. Cost containment in the clinical laboratory-to be or seem to be, or when is a non-test a test? JAMA 1980;243:1659.

${ }^{9}$ Winkelman JW. Less utilisation of the clinical laboratory produces disproportionately small true cost reductions. Hum Pathol 1984:15:499-501.

${ }^{10}$ Winkelman JW, Hill RB. Clinical laboratory responses to reduced funding. JAMA 1984;252:2435-40.

Requests for reprints to: Mr IF Tarbit, Department of Clinical Biochemistry, Freeman Hospital, Freeman Road, High Heaton, Newcastle upon Tyne N37 7DN, England. 